\title{
Macular and peripapillary vessel density measurement in the evaluation of Non-proliferative Diabetic Retinopathy
}

\section{Dawei Yang}

Department of Ophthalmology, Guangdong General Hospital, Guangdong Academy of Medical Sciences

\section{Dan Cao}

Guangdong General Hospital, Guangdong Academy of Medical Sciences

\section{Honghua Yu}

Guangdong General Hospital, Guangdong Academy of Medical Sciences

\section{Xiaohong Yang}

Guangdong General Hospital, Guangdong Academy of Medical Sciences

\section{Zhongning Huang}

Guangdong General Hospital, Guangdong Academy of Medical Sciences

\section{Yunkao Zeng}

1. Guangdong General Hospital, Guangdong Academy of Medical Sciences, Department of Ophthalmology; 2. Shantou University Medical College

\section{Xuenan Zhuang}

1. Guangdong General Hospital, Guangdong Academy of Medical Sciences, Department of Ophthalmology; 2. Shantou University Medical College

\section{Qiaowei Wu}

1.Guangdong General Hospital, Guangdong Academy of Medical Sciences, Department of Ophthalmology; 2.Southern Medical University

\section{Baoyi Liu}

1.Guangdong General Hospital, Guangdong Academy of Medical Sciences, Department of Ophthalmology; 2.Southern Medical University

\section{Liang Zhang ( $\nabla$ zhangliang5413@163.com )}

https://orcid.org/0000-0002-0454-8001

\section{Research article}

Keywords: diabetic retinopathy, optical coherence tomography angiography, vessel density, RNFL thickness, FD 300 
DOI: https://doi.org/10.21203/rs.2.141/v2

License: (c) (1) This work is licensed under a Creative Commons Attribution 4.0 International License. Read Full License 


\section{Abstract}

Purpose: To compare vessel density in macular and peripapillary area between control subjects and patients with non-proliferative diabetic retinopathy (NPDR) using optical coherence tomography angiography (OCTA) and to evaluate the association between RNFL thickness and different stage of diabetic retinopathy. Methods: A total of 170 eyes (normal control, 43; mild NPDR, 43; moderate NPDR, 42; severe NPDR, 42) underwent OCTA imaging. Optical coherence tomography angiographic parameters were vessel densities of superficial capillary plexus (SCP), deep capillary plexus (DCP) in macular area and peripapillary area. Results: Vessel density of SCP and DCP in macular area, peripapillary area as well as RNFL thickness were $53.13 \% \pm 3.03 \%, 52.07 \% \pm 2.28 \%, 53.78 \% \pm 3.66 \%$ and $128.86 \mu \mathrm{m} \pm 11.32 \mu \mathrm{m}$, respectively, in control subjects; $50.24 \% \pm 3.81 \%, 47.40 \% \pm 45.02 \%, 46.50 \% \pm 6.03 \%$ and $124 \mu \mathrm{m} \pm 13.97 \mu \mathrm{m}$, respectively, in mild NPDR; $46.80 \% \pm 5.23 \% ; 44.39 \% \pm 3.99 \% ; 44.64 \% \pm 4.23 \% ; 121.02 \mu \mathrm{m} \pm 20.86 \mu \mathrm{m}$, respectively, in moderate NPDR; $42.82 \% \pm 5.46 \%, 42.34 \% \pm 5.14 \%, 43.16 \% \pm 4.47 \%, 118.60 \mu \mathrm{m} \pm 21.91 \mu \mathrm{m}$ in severe NPDR. The reduction of vessel density of SCP and DCP in macular area, peripapillary area as well as RNFL thickness were correlated with increasing severity of DR. Vessel density of SCP and DCP in macular area, peripapillary area and foveal density 300 (FD 300) in normal control were significantly higher than that of mild, moderate and severe NPDR groups. (all $\mathrm{P}<0.001)$. Vessel density of DCP shows better ability to identify the severity of $\mathrm{DR}(0.913 ; 95 \% \mathrm{Cl}=0.867-0.958$; cut off value:0.75) than FD 300 , vessel density of SCP in macular area and peripapillary area. Conclusion: Macular and peripapillary vessel density as well as RNFL thickness decreased as DR progresses. Vessel density in DCP could be an objective and sensitive indicator for monitoring progression of DR. OCTA might be clinically useful to evaluate microvascular and microstructural alterations in macula and optive nerve head (ONH), thus providing a new method to study the course of DR.

\section{Introduction}

Diabetic retinopathy is the leading cause of blindness in economically active people worldwide and also known as a microvascular complication of diabetes mellitus.[1, 2]

Traditionally, fluorescein angiography (FA) has been used for the diagnosis and classification of DR even it has a number of potential side effects. [3, 4] Optical coherence tomography angiography (OCTA) is an alternative non-invasive angiographic technique which can demonstrate a 3-dimensional non-invasive vascular mapping at the microcirculation level.[5] With utilizing of OCTA, visualization of morphology of retinal and choroidal vessels and identification of ischemic area on a layer-by-layer basis all became possible[6]. Previous researches have used OCTA to indicate that vessel density in capillary plexus and choriocapillaries reduced as DR progressed[7-9]. However, more and more research has indicated DR as a neurovascular disease as evidence showed prominent decreased RNFL thickness in early stage of DR[10, 11]. Since optic nerve head (ONH) microcirculation as well as RNFL thickness can now be easily demonstrated as well as quantified at present, it would be meaningful to analyze the neurovascular alteration in optic disc as well as in macular area in diabetic patients. Although OCTA has been used to conduct studies towards retina and optic disc[12-14], seldom did studies combine these two areas in one 
research. Actually, it should be cautioned when investigating DR patients with glaucoma, or DR patients with other fundus disease that might affect macular perfusion. Simply depending on vessel density of either one might cause bias. Hence, measuring vessel density of both optic disc and macular area might provide a more comprehensive assessment on DR patients even both microcirculation origin from different sources. In this study, we aim to investigate macular and peripapillary vessel density, and RNFL thickness between normal subjects and diabetic patients with different stages of non-proliferative diabetic retinopathy (NPDR) using OCTA and to analyze the relation among these indicators.

\section{Methods}

\section{Subjects}

We recruited patients with type 2 diabetes mellitus (T2DM) and healthy control subjects who presented between January 2017 to January 2018 from Guangdong General Hospital. Written informed consent was obtained from all participants. A single eye was randomly selected if both were eligible. Exclusion criteria for all participants were as follows: any other ocular disease that may affect ocular circulation (e.g. glaucoma, age-related macular degeneration, retinal vascular occlusion, refractive error $>3$ diopters[D]), intraocular surgery, hypertension exceeding $150 / 100 \mathrm{mmHg}$, intraocular pressure (IOP) $>21 \mathrm{mmHg}$. Individuals with a family history of glaucoma were also excluded in the present study. All participants were tested for best IOP, corrected visual acuity (BCVA), and refractive error (autorefractometry). Slit-lamp and fundus examinations using direct and/or indirect ophthalmoscope were performed. ETDRS 35 degree 7-standard fields color retinal photographs (Topcon TRC; Topcon, Tokyo, Japan) was required for each participant. DR was graded according to the International Diabetic Retinopathy Severity Scale.

Fundus photographs evaluation and stage classification were done by two experienced graders (DC and $\mathrm{ZNH})$.

\section{Optical coherence tomography angiogram acquisition and analysis}

Optical coherence tomography angiography was performed using AngioVue Optical coherence tomography angiography system (RTVue-XR Avanti; Optovue, Fremont, CA, USA, version 2017.1.0.151). Each subject underwent two imaging sections that include a $6 \times 6-\mathrm{mm}$ region centered in the fovea and a $4.5 \times 4.5$-mm scan centered in the optic disc. Split-spectrum amplitude decorrelation angiography (SSADA) software algorithm was used for computing a flow map of each scan. Motion Correction Technology of Optovue software was used to compensate for motion artifacts. Imagine with inadequate

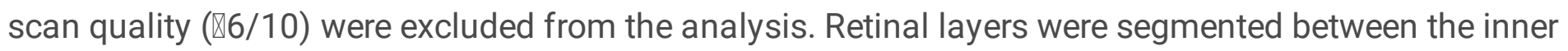
limiting membrane and the retinal pigment epithelium on the basis of the optical coherence tomography structural image. Segmentation of the angiogram would be checked to avoid analysis error. An en face optical coherence tomography angiogram was produced by maximum decorrelation projection of the segmented retina. 
The software automatically segmented the tissue into the macula (superficial, deep, outer retina, and choriocapillaries) and optic nerve head (optic nerve head, vitreous, radial peripapillary capillary, and choroid). Macular vessel density parameters were measured in the superficial and deep retinal optical coherence tomography angiogram of the macula. The peripapillary vessel density was measured for the radial peripapillary capillary (Fig1).

The boundaries were as follows: a slab extending from the internal limiting membrane to $10 \mu \mathrm{m}$ above inner plexiform layer was generated for detecting the vessel density of superficial capillary plexus. A slab extending from $10 \mu \mathrm{m}$ above inner plexiform layer to $10 \mu \mathrm{m}$ below the outer plexiform layer for the measurement of vessel density of deep capillary plexus and a slab extending from inner limiting membrane to retinal nerve fiber layer for the measurement of peripapillary vessel density.

Peripapillary region was divided into 8 sectors (Fig 1a), designated as nasal superior, nasal inferior, inferior nasal, inferior tempo, tempo inferior, tempo superior, superior tempo and superior nasal. Foveal avascular zone area ( $\mathrm{mm} 2)$ evaluated in the superficial plexus was identified as non-flow area and measured by the built-in software that delineated the segment of the foveal avascular zone automatically. Foveal thickness was calculated from the mean central point of fovea from inner limiting membrane to retinal pigment epithelium. Foveal density 300 (FD 300) is identified as the vessel density of the annular area surrounded by $300 \mu \mathrm{m}$ outwards with the FAZ inner boundary.

Two readers ( $\mathrm{DC}$ and $\mathrm{ZNH}$ ) evaluated the angiographic features on optical coherence tomography angiogram. Both readers were masked to the DR status. Image quality was considered by including images having Scan Quality of at least 6/10. In patients with poor images, we repeated the scans until an image with at least fair quality could be obtained.

\section{Statistical Analysis}

Statistical analysis was performed with SPSS 19.0 software (SPSS. Inc, Chicago, IL, USA). Qualitative variables are presented as number and percentage. Quantitative variables are presented as means and standard deviations. One-way analysis of variance test was used to compare the mean values of vessel density of superficial, deep and peripapillary area between groups. Receiver operator characteristic (ROC) curve was used to assess the relationship between the severity of DR and OCTA parameters and detect the sensitivity and specificity of the ROC curve. Criterion significance was assessed at the $P<0.05$ level.

This study has been performed in accordance with the Declaration of Helsinki and was approved by the Research Ethics Committee of Guangdong General Hospital (registration number: gdrec2016232A).

\section{Results}

\section{Demographics}

A total of 170 eyes of 43 normal controls and 127 eyes of 127 diabetic patients with NPDR (mild NPDR, 43; moderate NPDR, 42; severe NPDR, 42) were assessed initially for image quality after they met 
inclusion and exclusion criteria described in methods. Baseline demographics of the entire cohort were comparable in all 4 groups (Table 1). Of the 170 patients, 92 (54.1\%) were male and 78 (45.9\%) were female. Gender and age showed no significant differences in between-groups comparison. Of the 170 selected eyes, 88 (51.8\%) were right eyes, and 82 (48.2 \%) were left eyes. Patients were classified into normal group and NPDR (mild NPDR, moderate NPDR, severe NPDR) groups.

\section{Vessel density index and RNFL thickness measurement}

A series of color fundus photos and OCTA color-coded vascular vessel density maps listed as nonproliferative diabetic retinopathy progresses were shown in Fig 2. Details of vessel density in different layers of macular area, peripapillary area were illustrated in Table 2 and Fig 3. Vessel density in SCP and DCP of macular area, and peripapillary area were $(53.13 \% \pm 3.03 \%, 52.07 \% \pm 2.28 \%$ and $53.78 \% \pm 3.66 \%)$ in control subjects, $(50.24 \% \pm 3.81 \%, 47.40 \% \pm 5.02 \%$ and $46.50 \% \pm 6.03 \%)$ in mild NPDR, $(46.80 \% \pm 5.23 \%$; $44.39 \% \pm 3.99 \%$ and $44.64 \% \pm 4.23 \%)$ in moderate NPDR, $(42.82 \% \pm 5.46 \%, 42.34 \% \pm 5.14 \%$ and $43.16 \%$ $\pm 4.47 \%$ ) in severe NPDR. Vessel density in SCP and DCP of macular area as well as vessel density in peripapillary area showed significant decrease as the severity of DR progresses.

Vessel density in different quadrants of peripapillary area and RNFL thickness were also shown in Table 2. Significant differences in between-groups comparison were found in the vessel density at all quadrants $(\mathrm{p} \otimes 0.05)$. In the present study, peripapillary vessel density was significantly correlated with vessel density of SCP and DCP (Fig.4). In terms of RNFL thickness, significant difference was also found in comparison among groups $(p=0.03)$.

\section{AUROC analysis}

Table 3 detailed the performance of FD 300, vessel density of SCP and DCP in macular area, and peripapillary area for identifying severity of DR based on AUROC curve. AUC in FD300, SCP and DCP of macular area and peripapillary area and were $83.5 \%, 90.5 \%, 91.3 \%$ and $93.0 \%$, respectively. Vessel density of DCP shows better ability to identify the severity of $\mathrm{DR}(0.913 ; 95 \% \mathrm{Cl}=0.867-0.958$; cut off value:0.75) than in FD 300, vessel density of SCP in macular area and peripapillary area (Fig. 5).

\section{Discussion}

In the present study, as DR progressed, the reduction in vessel density of macular area and peripapillary area as well as RNFL thickness were noted. As for peripapillary area, vessel density from different quadrants all decreased and was significantly related to the scale of DR. In terms of AUROC analysis, vessel density of DCP in macular area showed better ability to identify the severity of NPDR when compared to vessel density of SCP, RPC density and FD300.

The pathology of DR remained as the object of conjecture and hypotheses. Traditionally, DR has been considered as one of the microvascular diabetic complications and can generally be described as progressing through two stages, including NPDR and proliferative DR[15]. Hyperglycemia was considered 
to be an important factor in the etiology of DR and initiates downstream events such as basement membrane thickening, pericyte apoptosis and retinal capillary non-vessel density[16, 17]. Inflammation, endoplasmic reticulum function disorders and elevated level of reactive oxygen species (ROS) are major causative factors involved in the pathogenesis of DR which can finally lead to vessel density problems in retina as well as $\mathrm{ONH}[18-20]$. In our research, we documented the reduction in the macular vessel density and peripapillary vessel density was also significantly related to the degree of DR scale. In the analysis of different quadrants of peripapillary area, vessel density from all quadrants of peripapillary area decreased as DR progressed. Our results indicated that the reduction of $\mathrm{ONH}$ microcirculation in diabetic eyes was prominent. Alteration of peripapillary vessel density could be another sensitive indicator for the progression of DR.

In NPDR, neurodegeneration was also noted for decades. Histologically, $\mathrm{ONH}$ is supplied by two main source of blood flow: the superficial layers of the optic nerve head (nerve fiber layer on the surface of the optic disc) by the central retinal artery (CRA) circulation; and the deeper layers (the prelaminar, lamina cribrosa, and retrolaminar regions) by the posterior ciliary artery (PCA) circulation[21, 22]. The radial peripapillary capillaries (RPCs) are located in the inner part of the nerve fiber layer. They are parallel to the retinal ganglion cell axons and arched up steeply to supply superficial RNFL around the ONH[23, 24]. Studies indicated that when neuron activity increased locally in visual stimulus, retinal arterioles dilated to ensure adequate blood supply for actively firing neurons[25]. The regulation of blood flow was considered in response to neuroactivity. However, recent research has pointed out that retinal neurodegeneration might precede microvascular dysfunction in DR.[26-29]. Many researches have suggested neuronal loss in the inner retina of DR eyes by demonstrating the reduction of nerve fiber, ganglion cell and plexiform layers using optical coherence tomography, indicating retinal neurodegeneration is an early component of DR, which can precede visible vasculopathy. Also, Studies have found out that RNFL thinning correlated positively with severity of DR[30]. In the present research, we also documented significant difference in average RNFL thickness in-between group comparison. RNFL thickness reduced as the severity of DR increased. Our understanding of the pathophysiology process of DR has been revolutionized by decades of research. Indeed, DR is now more accurately defined as a neurovascular rather than a microvascular disease. Further studies is definitely required for the clarification of which one trigger the occurrence of DR.

According to the previous research, the vascular networks become more complex with multiple overlapping capillary beds away from the foveal center. It has raised the concern that OCTA may not be able to display some of the finer capillary structure, which might influence the accuracy of vessel densities[31]. The alteration of foveal avascular zone, such as capillary drop out around FAZ area, has even been identified in patients with early stage of DR[32-34]. FD 300 is identified as the vessel density of the annular area surrounded by $300 \mu \mathrm{m}$ outwards with the FAZ inner boundary. It is reasonable to consider vasculature in FD300 was organized into less layers and FD300 as another sensitive indicators for the severity of DR. Thus, we intend to use FD 300 to predict the progress of NPDR in the present study. However, AUROC analysis showed that vessel density of DCP shows better ability to identify the severity 
of DR than other vessel density index. It is still of great interest to analyze FD 300 in different fundus diseases.

Indeed, related literature has shown that the reduction of macular vessel density is in accordance with the progression of DR and others researches have been conducted to observe the morphology neovascularization of the disc $[13,14,35]$. Compared with previous researches, the present study has several strengths. Firstly, the decease of vessel density and RNFL thickness are notable in the current research. Since the initial course of DR are still controversial now, the current study might have provided a new insight in the occurrence and development of DR in human using OCTA. Also, our results might imply that therapeutic strategies targeting signaling pathways that cause microvascular dysfunction and retinal neurodegeneration are both important in preventing the development of DR. Hence, we probably should pay equal attention to the intervention for arresting retinal vasculopathy and neurodegeneration. Secondly, patients with diabetic optic neuropathy (DON) can take place in all stages of DR. The current research revealed the reduction of RNFL thickness as DR progressed, which might be consistent with the hypothesis that NAION might have a devastating effect on the integrity of the optic nerve followed by RNFL loss[36-38]. However, this result requires more prospective studies to verify. Last but not the least, this research combines both macular and peripapillary vessel density in one cross-sectional DR study, providing a comprehensive overview of diabetic retinal and optic papillary changes.

Nevertheless, there are limitations of the current study. GCC measurement was not taken into account in the present research. Further study would be highly recommended to take this factor into consideration. Also, we acknowledge that this is merely an extrapolation of our results, a longitudinal study should be further conducted before such conclusion could be verified.

In conclusion, our results indicated that as DR progressed, the reduction of vessel density in macular and peripapillary area as well as RNFL thickness were prominent. Vessel density of DCP in macular area showed good ability to identify the severity of NPDR. With regard to the optic nerve, microvascular insufficiency and RNFL defects were noted distinctly. OCTA might be a good tool for the pathophysiological investigation for DR since OCTA can quantify vessel density and neurodegenerative changes in subjects with diabetes.

\section{Abbreviations}

NPDR: non-proliferative diabetic retinopathy; OCTA: optical coherence tomography angiography; SCP: superficial capillary plexus, DCP: deep capillary plexus; RNFL: retinal never fiber layer; FA: fluorescein angiography; ONH: optic nerve head; T2DM: type 2 diabetes mellitus; BCVA: best corrected visual acuity; SSADA: Split-spectrum amplitude decorrelation angiography; RPC: radial peripapillary capillary; NS: nasal superior, NI: nasal inferior, IN: inferior nasal, IT: inferior tempo, TI: tempo inferior, TS: tempo superior; ST: superior tempo; SN: superior nasal; ILM: inner limiting membrane; FAZ: foveal avascular zone area; ROS: reactive oxygen species; CRA: central retinal artery; PCA: posterior ciliary artery; DON: diabetic optic neuropathy; 


\section{Declarations}

\section{Ethics approval and consent to participate}

This study has been performed in accordance with the Declaration of Helsinki and was approved by the Research Ethics Committee of Guangdong General Hospital (registration number: gdrec2016232A).

\section{Consent to publish}

Written informed consents for publication of the clinical details and accompanying images were obtained from the patients. A copy of the written consents is available for review by the Editor of this journal.

\section{Availability of data and materials}

All data generated or analyzed of this study are included in this article. Raw and processed data during the current study are available from the corresponding author upon reasonable request.

\section{Competing interests}

The authors declared no conflicts of interest to this work

\section{Funding}

National Natural Science Foundation of China/Grant Number:81870663

Guangzhou Science and Technology Program/Grant Number:201607010343

The funding body had no role in the design or conduct of this research.

\section{Authors' contributions}

Conception and design of the study: LZ, DC, HHY, XHY;

Acquisition of data: DWY, YKZ, XNZ, BYL, QWW.

Analysis and interpretation of data: DWY, ZNH, YKZ

Drafting of the manuscript: DWY, XNZ

Critically revision of the manuscript: LZ, DC, HHY 
Provision of materials, patients or resources: LZ, HHY, ZNH

All authors read and approved the final version to be published

\section{Acknowledgement}

Not applicable.

\section{References}

1. Cheung N, Mitchell P, Wong TY: Diabetic retinopathy. Lancet 2010, 376(9735):124-136.

2. Yau JW, Rogers SL, Kawasaki R, Lamoureux EL, Kowalski JW, Bek T, Chen SJ, Dekker JM, Fletcher A, Grauslund J et al: Global prevalence and major risk factors of diabetic retinopathy. Diabetes care 2012, 35(3):556-564.

3. Almalki WH, Abdalla AN, Elkeraie AF, Abdelhadi AM, Elrggal M, Elrggal ME: Effect of fluorescein angiography on renal functions in type 2 diabetes patients: A pilot study. Saudi journal of kidney diseases and transplantation : an official publication of the Saudi Center for Organ Transplantation, Saudi Arabia 2017, 28(3):491-498.

4. Alemzadeh-Ansari MJ, Beladi-Mousavi SS, Feghhei M: Effect of fluorescein on renal function among diabetic patients. Nefrologia : publicacion oficial de la Sociedad Espanola Nefrologia 2011, 31(5):612-613.

5. Vujosevic S, Midena E: Retinal layers changes in human preclinical and early clinical diabetic retinopathy support early retinal neuronal and Muller cells alterations. Journal of diabetes research 2013, 2013:905058.

6. Ishibazawa A, Nagaoka T, Takahashi A, Omae T, Tani T, Sogawa K, Yokota H, Yoshida A: Optical Coherence Tomography Angiography in Diabetic Retinopathy: A Prospective Pilot Study. American journal of ophthalmology 2015, 160(1):35-44 e31.

7. Hasegawa N, Nozaki M, Takase N, Yoshida M, Ogura Y: New Insights Into Microaneurysms in the Deep Capillary Plexus Detected by Optical Coherence Tomography Angiography in Diabetic Macular Edema. Investigative ophthalmology \& visual science 2016, 57(9):OCT348-355.

8. Agemy SA, Scripsema NK, Shah CM, Chui T, Garcia PM, Lee JG, Gentile RC, Hsiao YS, Zhou Q, Ko T et al: Retinal Vascular Perfusion Density Mapping Using Optical Coherence Tomography Angiography In Normals And Diabetic Retinopathy Patients. Retina 2015, 35(11):2353-2363.

9. Hwang TS, Jia Y, Gao SS, Bailey ST, Lauer AK, Flaxel CJ, Wilson DJ, Huang D: Optical Coherence Tomography Angiography Features Of Diabetic Retinopathy. Retina 2015, 35(11):2371-2376.

10. Srinivasan S, Dehghani C, Pritchard N, Edwards K, Russell AW, Malik RA, Efron N: Corneal and Retinal Neuronal Degeneration in Early Stages of Diabetic Retinopathy. Investigative ophthalmology \& visual science $2017,58(14): 6365-6373$. 
11. Shi R, Guo Z, Wang F, Li R, Zhao L, Lin R: Alterations in retinal nerve fiber layer thickness in early stages of diabetic retinopathy and potential risk factors. Current eye research 2018, 43(2):244-253.

12. Alnawaiseh M, Brand C, Lauermann JL, Eter $\mathrm{N}$ : [Flow density measurements using optical coherence tomography angiography : Impact of age and gender]. Ophthalmologe 2018, 115(8):659-662.

13. AttaAllah HR, Mohamed AAM, Ali MA: Macular vessels density in diabetic retinopathy: quantitative assessment using optical coherence tomography angiography. International ophthalmology 2018.

14. Akiyama H, Li D, Shimoda Y, Matsumoto H, Kishi S: Observation of neovascularization of the disc associated with proliferative diabetic retinopathy using OCT angiography. Japanese journal of ophthalmology 2018, 62(3):286-291.

15. Lutty GA: Effects of diabetes on the eye. Investigative ophthalmology \& visual science 2013, 54(14):ORSF81-87.

16. Calderon GD, Juarez OH, Hernandez GE, Punzo SM, De la Cruz ZD: Oxidative stress and diabetic retinopathy: development and treatment. Eye 2017, 31(8):1122-1130.

17. Kowluru RA, Kowluru A, Mishra M, Kumar B: Oxidative stress and epigenetic modifications in the pathogenesis of diabetic retinopathy. Progress in retinal and eye research 2015, 48:40-61.

18. Kowluru RA, Tang J, Kern TS: Abnormalities of retinal metabolism in diabetes and experimental galactosemia. VII. Effect of long-term administration of antioxidants on the development of retinopathy. Diabetes 2001, 50(8):1938-1942.

19. Kanwar M, Chan PS, Kern TS, Kowluru RA: Oxidative damage in the retinal mitochondria of diabetic mice: possible protection by superoxide dismutase. Investigative ophthalmology \& visual science 2007, 48(8):3805-3811.

20. Song S, Tan J, Miao Y, Li M, Zhang Q: Crosstalk of autophagy and apoptosis: Involvement of the dual role of autophagy under ER stress. Journal of cellular physiology 2017, 232(11):2977-2984.

21. Hayreh SS: Blood supply of the optic nerve head and its role in optic atrophy, glaucoma, and oedema of the optic disc. The British journal of ophthalmology 1969, 53(11):721-748.

22. Hayreh SS: Posterior ciliary artery circulation in health and disease: the Weisenfeld lecture. Investigative ophthalmology \& visual science 2004, 45(3):749-757; 748.

23. Mansoori T, Sivaswamy J, Gamalapati JS, Balakrishna N: Topography and correlation of radial peripapillary capillary density network with retinal nerve fibre layer thickness. International ophthalmology 2018, 38(3):967-974.

24. Scoles D, Gray DC, Hunter JJ, Wolfe R, Gee BP, Geng Y, Masella BD, Libby RT, Russell S, Williams DR et al: In-vivo imaging of retinal nerve fiber layer vasculature: imaging histology comparison. BMC ophthalmology 2009, 9:9.

25. Kur J, Newman EA, Chan-Ling T: Cellular and physiological mechanisms underlying blood flow regulation in the retina and choroid in health and disease. Progress in retinal and eye research 2012, 31(5):377-406. 
26. Parisi V, Uccioli L, Parisi L, Colacino G, Manni G, Menzinger G, Bucci MG: Neural conduction in visual pathways in newly-diagnosed IDDM patients. Electroencephalography and clinical neurophysiology 1998, 108(5):490-496.

27. Stem MS, Gardner TW: Neurodegeneration in the pathogenesis of diabetic retinopathy: molecular mechanisms and therapeutic implications. Current medicinal chemistry 2013, 20(26):3241-3250.

28. Verma A, Rani PK, Raman R, Pal SS, Laxmi G, Gupta M, Sahu C, Vaitheeswaran K, Sharma T: Is neuronal dysfunction an early sign of diabetic retinopathy? Microperimetry and spectral domain optical coherence tomography (SD-OCT) study in individuals with diabetes, but no diabetic retinopathy. Eye 2009, 23(9):1824-1830.

29. van Dijk HW, Verbraak FD, Kok PH, Stehouwer M, Garvin MK, Sonka M, DeVries JH, Schlingemann RO, Abramoff MD: Early neurodegeneration in the retina of type 2 diabetic patients. Investigative ophthalmology \& visual science 2012, 53(6):2715-2719.

30. Srivastav K, Saxena S, Mahdi AA, Kruzliak P, Khanna VK: Increased serum urea and creatinine levels correlate with decreased retinal nerve fibre layer thickness in diabetic retinopathy. Biomarkers : biochemical indicators of exposure, response, and susceptibility to chemicals 2015, 20(6-7):470-473.

31. Mo S, Krawitz B, Efstathiadis E, Geyman L, Weitz R, Chui TY, Carroll J, Dubra A, Rosen RB: Imaging Foveal Microvasculature: Optical Coherence Tomography Angiography Versus Adaptive Optics Scanning Light Ophthalmoscope Fluorescein Angiography. Investigative ophthalmology \& visual science 2016, 57(9):OCT130-140.

32. Ashraf M, Nesper PL, Jampol LM, Yu F, Fawzi AA: Statistical Model of Optical Coherence Tomography Angiography Parameters That Correlate With Severity of Diabetic Retinopathy. Investigative ophthalmology \& visual science 2018, 59(10):4292-4298.

33. Cao D, Yang D, Huang Z, Zeng Y, Wang J, Hu Y, Zhang L: Optical coherence tomography angiography discerns preclinical diabetic retinopathy in eyes of patients with type 2 diabetes without clinical diabetic retinopathy. Acta diabetologica 2018, 55(5):469-477.

34. Johannesen SK, Viken JN, Vergmann AS, Grauslund J: Optical coherence tomography angiography and microvascular changes in diabetic retinopathy: a systematic review. Acta ophthalmologica 2018.

35. Hassan M, Sadiq MA, Halim MS, Afridi R, Soliman MK, Sarwar S, Agarwal A, Do DV, Nguyen QD, Sepah YJ: Evaluation of macular and peripapillary vessel flow density in eyes with no known pathology using optical coherence tomography angiography. Int J Retina Vitreous 2017, 3:27.

36. Bellusci C, Savini G, Carbonelli M, Carelli V, Sadun AA, Barboni P: Retinal nerve fiber layer thickness in nonarteritic anterior ischemic optic neuropathy: OCT characterization of the acute and resolving phases. Graefe's archive for clinical and experimental ophthalmology = Albrecht von Graefes Archiv fur klinische und experimentelle Ophthalmologie 2008, 246(5):641-647.

37. Dotan G, Goldstein M, Kesler A, Skarf B: Long-term retinal nerve fiber layer changes following nonarteritic anterior ischemic optic neuropathy. Clinical ophthalmology 2013, 7:735-740.

38. Duman R, Yavas GF, Veliyev I, Dogan M, Duman R: Structural changes of macula and optic disk of the fellow eye in patients with nonarteritic anterior ischemic optic neuropathy. International 
ophthalmology 2018.

\section{Tables}

Table 1 Demographic and ocular characteristics of the participants in four groups.

$$
\text { Normal }
$$

$\mathrm{P}$ value

Mild NPDR Moderate NPDR Severe NPDR

\begin{tabular}{cccccc}
\hline Number of eyes & 43 & 43 & 42 & 42 & 0.218 \\
Gender (female: male) & $22: 21$ & $24: 19$ & $24: 18$ & $22: 20$ & 0.391 \\
Left/right eye & $23: 20$ & $21: 22$ & $21: 21$ & $23: 19$ & 0.323 \\
Age(years) & $42.6 \pm 8.2$ & $43.1 \pm 7.1$ & $42.4 \pm 10.1$ & $44.0 \pm 9.3$ & 0.107 \\
\hline
\end{tabular}

Table 2 Macular perfusion and peripapillary perfusion and RNFL thickness of subjects observed 


\begin{tabular}{|c|c|c|c|c|c|}
\hline & Normal & Mild NPDR & $\begin{array}{l}\text { Moderate } \\
\text { NPDR }\end{array}$ & $\begin{array}{l}\text { Severe } \\
\text { NPDR }\end{array}$ & $\mathrm{p}$ value \\
\hline Macular perfusion of SCP & $53.13 \pm 3.03$ & $50.24 \pm 3.81$ & $46.80 \pm 5.23$ & $42.82 \pm 5.46$ & $n$ \\
\hline Macular perfusion of DCP & $52.07 \pm 2.28$ & $47.40 \pm 5.02$ & $44.39 \pm 3.99$ & $42.34 \pm 5.14$ & p \\
\hline FD300 & $55.04 \pm 4.89$ & $49.09 \pm 4.04$ & $48.05 \pm 5.35$ & $45.91 \pm 7.92$ & $\mathrm{p}$ \\
\hline Peripapillary perfusion & $53.78 \pm 3.66$ & $46.50 \pm 6.03$ & $44.64 \pm 4.23$ & $43.16 \pm 4.47$ & p \\
\hline Nasal Superior & $48.07 \pm 3.01$ & $46.18 \pm 4.66$ & $43.23 \pm 5.20$ & $40.51 \pm 6.57$ & p \\
\hline Superior Nasal & $51.92 \pm 3.36$ & $48.10 \pm 5.84$ & $46.65 \pm 5.78$ & $40.40 \pm 8.28$ & p \\
\hline Nasal Inferior & $48.81 \pm 2.81$ & $44.89 \pm 4.73$ & $42.46 \pm 5.27$ & $38.42 \pm 5.58$ & $\begin{array}{l}\mathrm{p} \\
<0,1\end{array}$ \\
\hline Inferior Nasal & $51.85 \pm 3.93$ & $49.93 \pm 4.89$ & $46.99 \pm 5.34$ & $40.41 \pm 8.05$ & $\begin{array}{c}\mathrm{p} \\
<001\end{array}$ \\
\hline Inferior Tempo & $57.55 \pm 3.02$ & $56.81 \pm 4.58$ & $53.13 \pm 4.79$ & $47.63 \pm 9.72$ & $\begin{array}{l}\mathrm{p} \\
<0001\end{array}$ \\
\hline Temporal Inferior & $54.20 \pm 3.44$ & $51.32 \pm 3.96$ & $49.76 \pm 5.63$ & $45.67 \pm 7.62$ & $\begin{array}{l}\mathrm{p} \\
0001\end{array}$ \\
\hline Tempo Superior & $57.45 \pm 2.88$ & $54.70 \pm 4.32$ & $51.77 \pm 5.41$ & $46.95 \pm 8.09$ & p \\
\hline Superior Tempo & $55.94 \pm 3.72$ & $53.20 \pm 4.60$ & $51.64 \pm 5.60$ & $46.43 \pm 9.94$ & p \\
\hline $\begin{array}{l}\text { Peripapillary RNFL } \\
\text { thickness }(\mu \mathrm{m})\end{array}$ & $128.86 \pm 11.32$ & $124 \pm 13.97$ & $121.02 \pm 20.86$ & $118.60 \pm 21.91$ & $=0.03$ \\
\hline
\end{tabular}

Vessel density in SCP, DCP and peripapillary area were reported as percentage.

NPDR: Non-proliferative diabetic retinopathy; PDR: proliferative diabetic retinopathy SCP: superficial capillary plexus; DCP: deep capillary plexus

Table 3 Performance parameters for perfusion index for identifying severity of DR

\begin{tabular}{lcccc}
\hline & AUROC (95\% CI) & Sensitivity (\%) & Specificity (\%) & Youden Index \\
\hline VD in SCP in macular area & $0.913(0.867-0.958)$ & 83.1 & 85.2 & 0.683 \\
VD in DCP in macular area & $0.930(0.886-0.975)$ & 88.1 & 86.9 & 0.750 \\
VD in peripapillary area & $0.905(0.860-0.950)$ & 83.3 & 83.6 & 0.669 \\
FD 300 & $0.835(0.760-0.910)$ & 82.7 & 59.2 & 0.419 \\
\hline
\end{tabular}


VD: vessel density

\section{Figures}

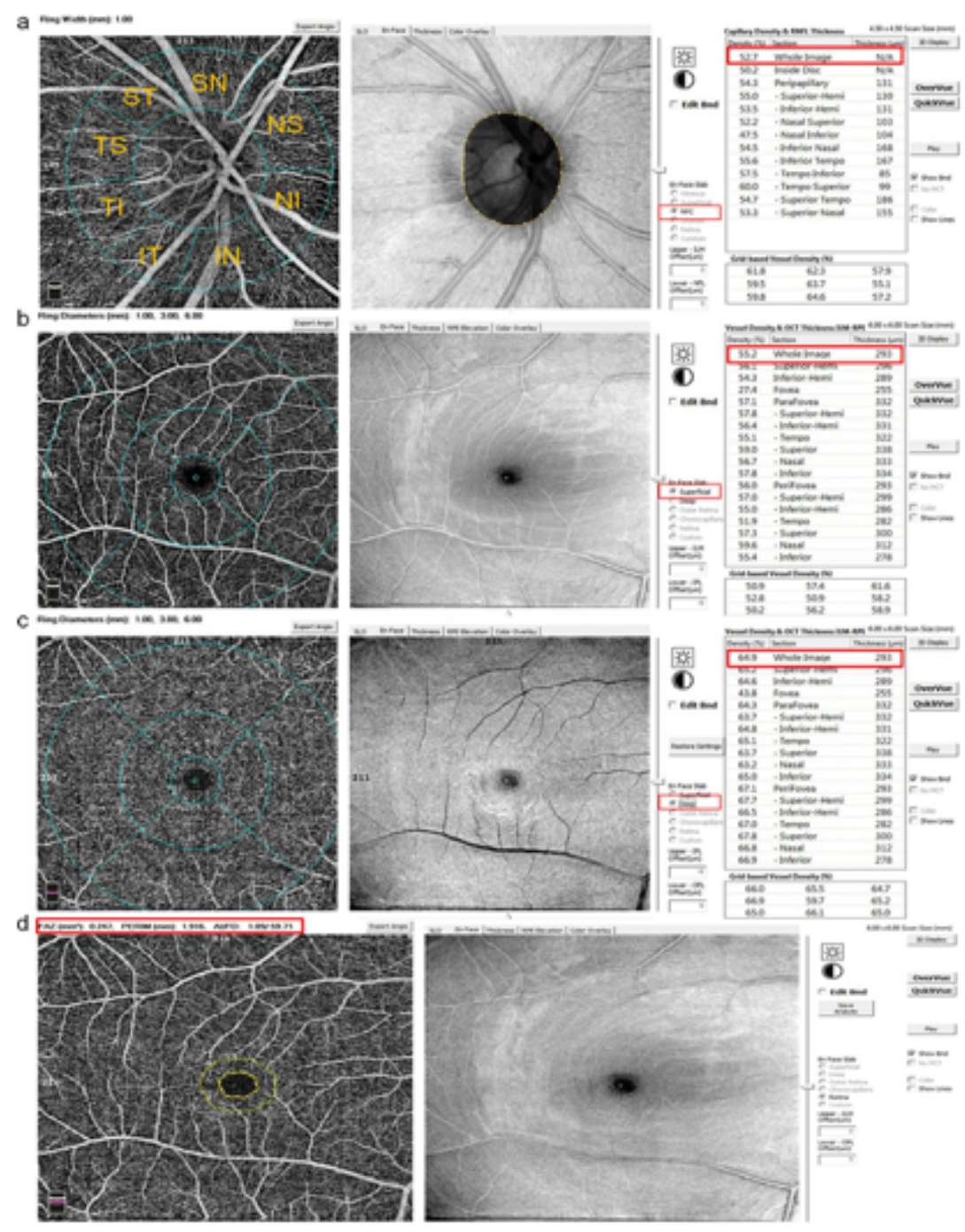

Figure 1

Example images of vessel density and RNFL measurement in a control subject (a. radial peripapillary capillaries; b. superficial capillary plexus; c. deep capillary plexus; d. quantification of FD300.). Vessel densities and RNFL thickness were calculated automatically (red box). 


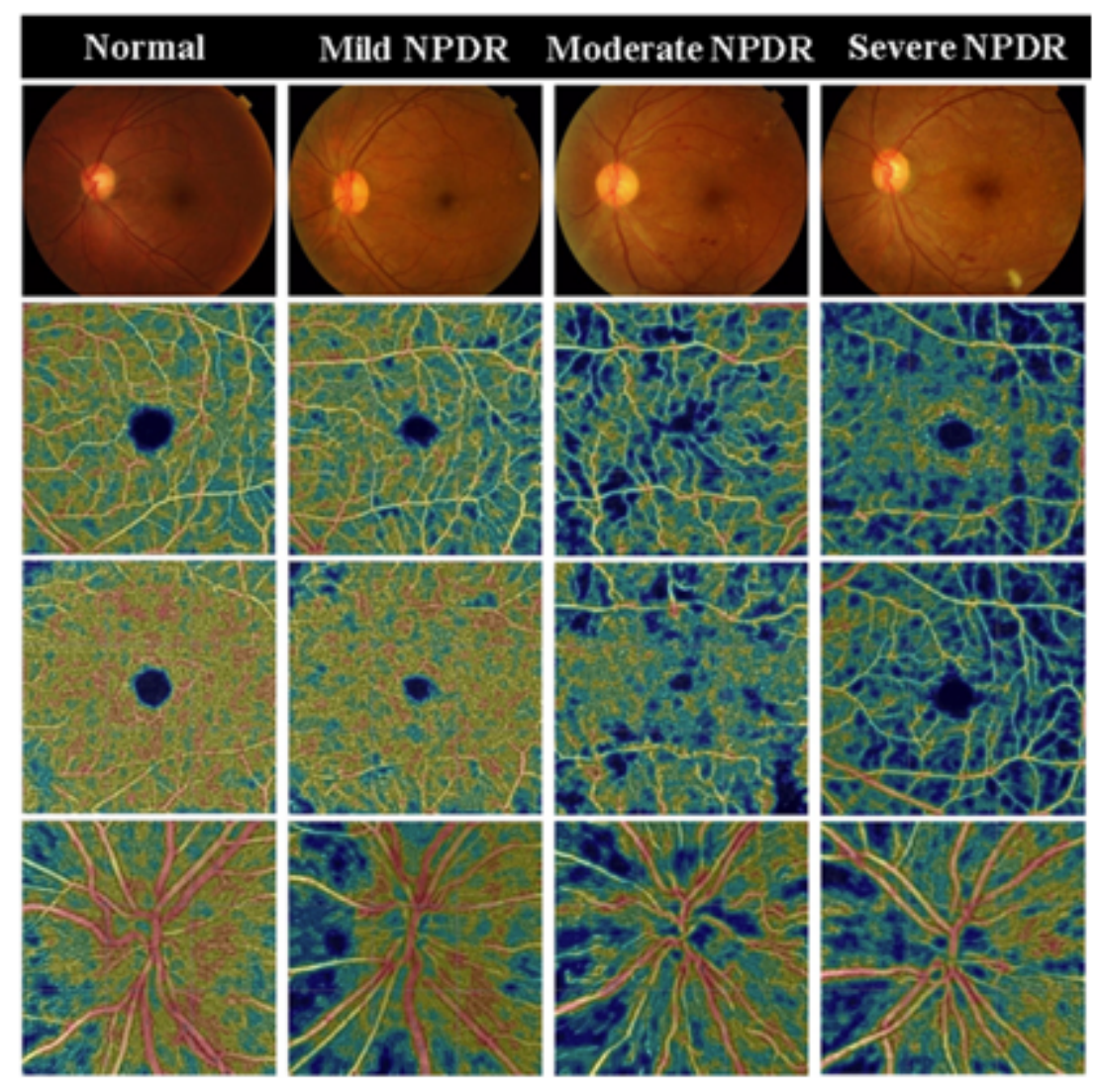

Figure 2

A series of color fundus photos (top row), SSADA OCTA color-coded vascular perfusion maps (second to the last row) demonstrating the perfusion density changes seen as non-proliferative diabetic retinopathy progresses. From left to right, we start with a normal eye and progress to mild NPDR, moderate NPDR, and finally severe NPDR. 

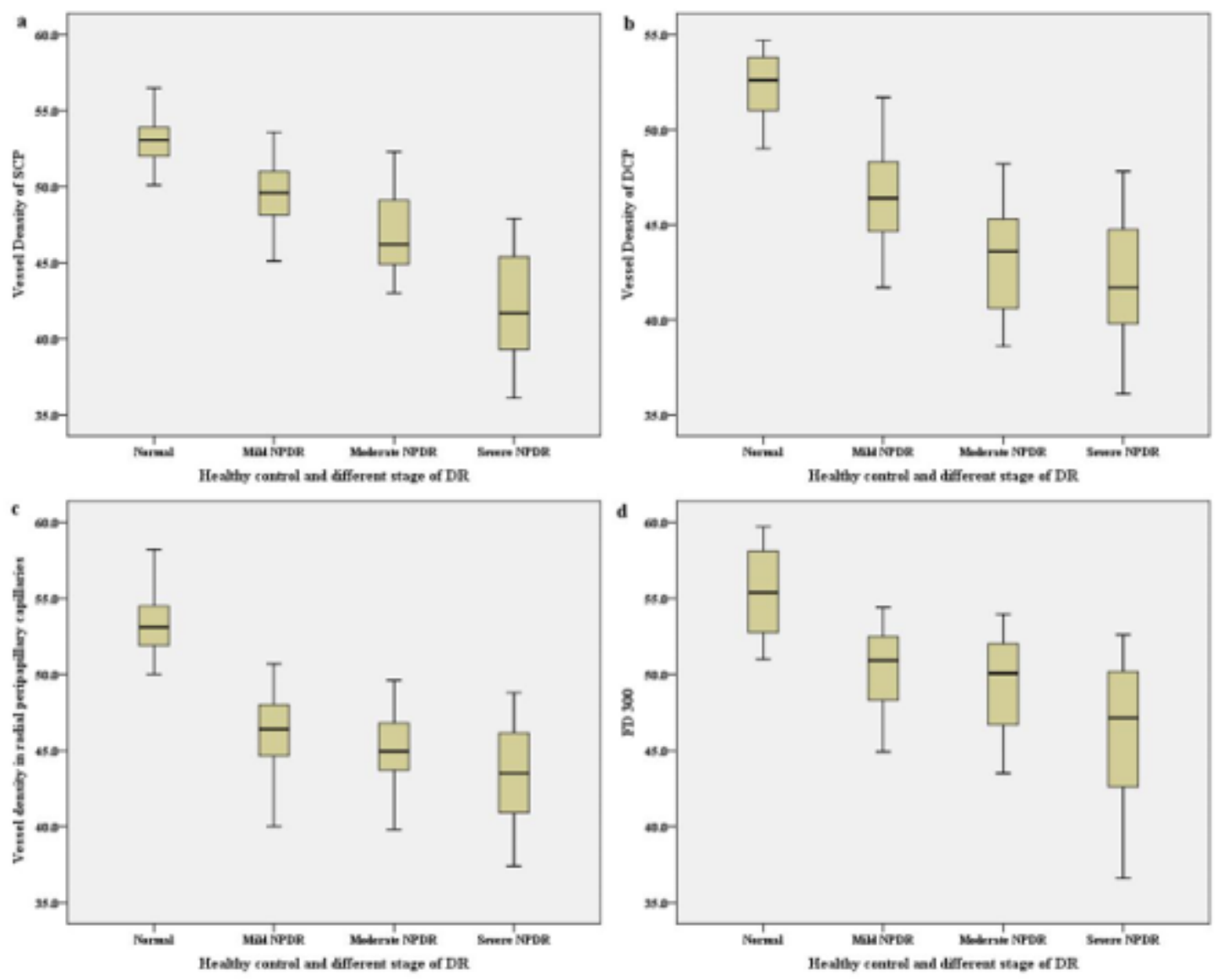

\section{Figure 3}

Box plots demonstrating the vessel density changes with normal control and different stage of DR.
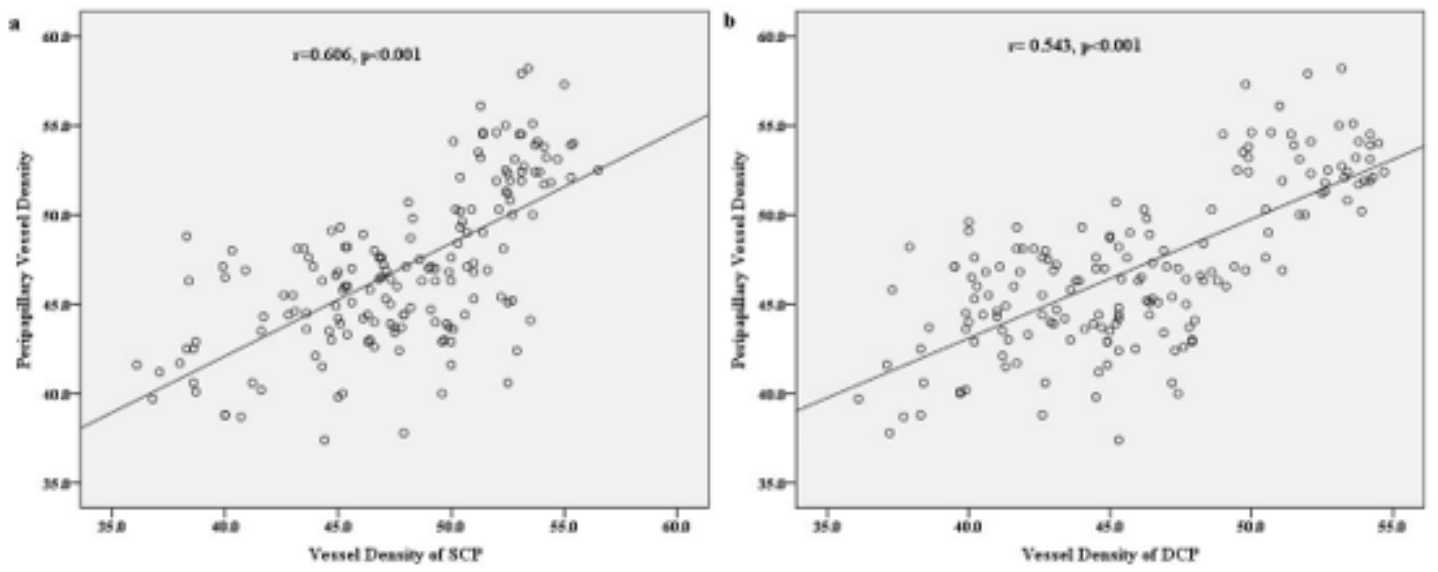

\section{Figure 4}

Correlation analyses between peripapillary vessel density and vessel density of SCP and DCP in macular area. Peripapillary vessel density was significantly correlated with vessel density of $\operatorname{SCP}(a)(r=0.606, p<$ $0.001)$ and vessel density of DCP $(d)(r=0.543, p<0.001)$ 


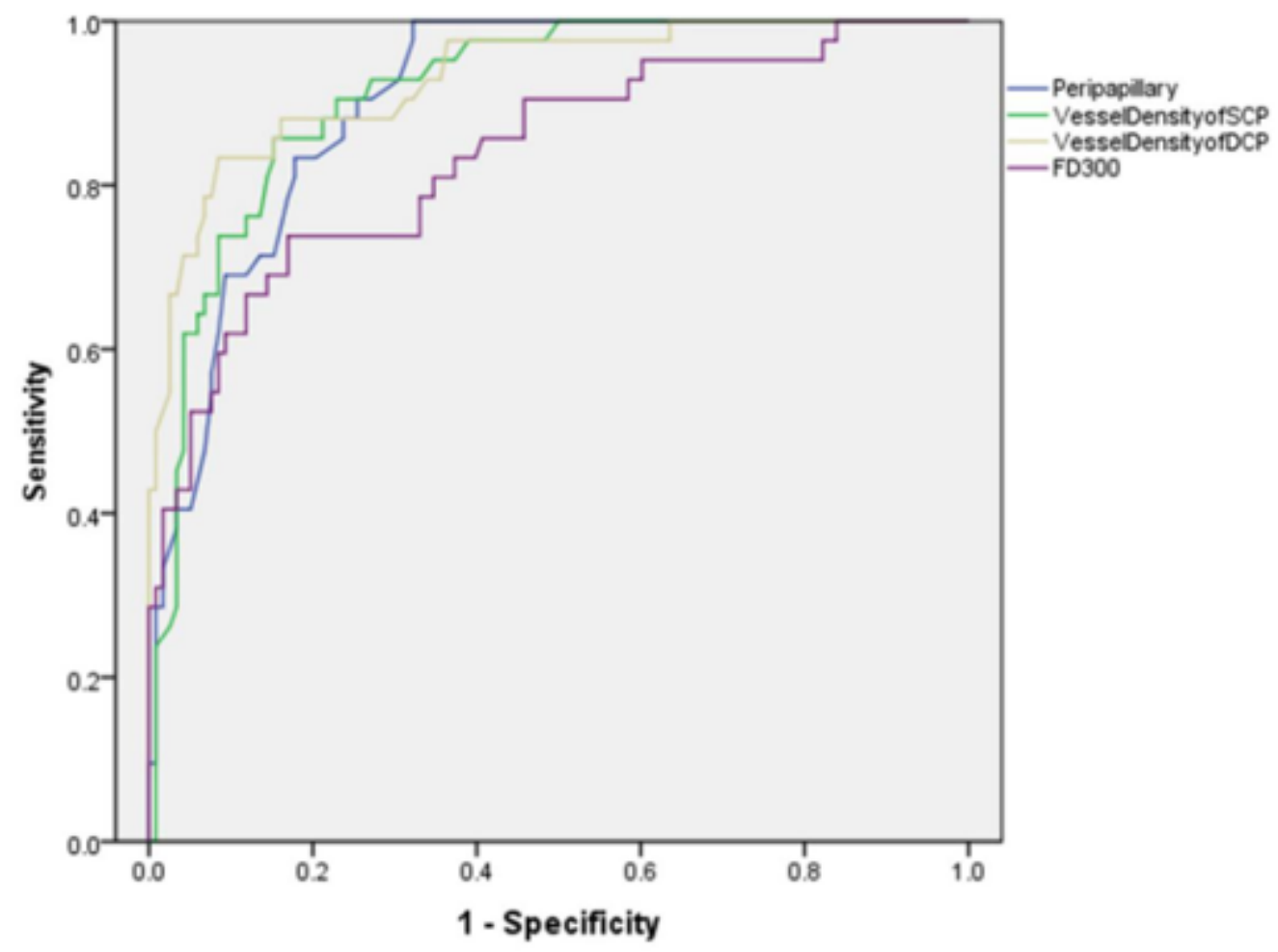

Figure 5

Receiver-operating characteristic (ROC) curves for SCP and DCP of macular area, and peripapillary area and FD 300 for detecting stage of DR. 